\title{
SURGICAL TREATMENT OF PRIMARY INTRA-ABDOMINAL COMPLICATIONS: ABSCESSES AND INFILTRATES
}

\author{
Skyba $V_{\bullet}^{1,3,4}$, Rybalchenko $V_{\bullet}^{2,3}$, Ivanko $0_{0^{1,3,4}}$, Voytyuk $N_{\bullet}^{1,3,4}$, Dar Yasin Akhmed \\ ${ }^{1}$ Bogomolets National Medical University, ${ }^{2}$ P.L. Shupyk National Medical Academy, \\ ${ }^{3}$ Kyiv Medical University of UAFM, ${ }^{4}$ Kyiv City Clinical Hospital No. 1
}

https://doi.org/10.35339/ic.8.1.30-36

\begin{abstract}
Purpose of the work. improving the results of surgical treatment of patients with primary intra-abdominal infiltrates and abscesses. Material and research methods. From 2006 to 2019, 191 patients with primary intra-abdominal infiltrates and abscesses were treated. The patients' age ranged from 16 to 85 years. There were 96 male patients $(50.26 \%), 95$ female patients $(49.74 \%)$. Results. The patients were divided into 3 subgroups depending on the underlying disease. The first group included $74(38.74 \%)$ patients with destructive appendicitis, of which $39(20.42 \%)$ were in the control group, and $35(18.32 \%)$ were studied. The second group included $48(25.13 \%)$ patients suffering from perforated gastric ulcer and 12 duodenal ulcer, of which the control group was $26(13.61 \%)$, and the studied group was $22(11.52 \%)$. The third group included $69(36.13 \%)$ patients with cholecystitis, of which $37(19.37 \%)$ were in the control group, and $32(16.76 \%)$ were studied. All patients were operated on. Conclusions. Surgical treatment is individualized depending on the disease, so with destructive appendicitis from 74 (38.74\%) laparotomic in 42 (21.99\%), laparoscopic in $32(16.75 \%)$, and in $12(6.28 \%)$ with conversion; perforated gastric ulcer and 12 duodenal ulcer in 48 (25.13\%) open laparotomy; with cholecystitis from $69(36.13 \%)$ in $48(25.13 \%)$ laparotomic and in $21(11.00 \%)$ laparoscopically. The use of water-jet technologies in 64 $(33.51 \%)$ patients made it possible to minimize damage to the serous membrane and cleanse the peritoneum from acquired formations.
\end{abstract}

Keywords. Abscesses and infiltrates of the abdominal cavity, surgical treatment.

\section{Introduction}

Today, urgent abdominal pathology is complicated by formation of abscesses and infiltrates in $20-25 \%$ of cases. Typically, the causes of intraabdominal formation can be acute appendicitis, acute cholecystitis, perforated gastric and duodenal ulcers, Meckel's diverticulum, as well as serious of other diseases [2]. Despite significant developments, these diseases do not tend to decrease, on the contrary, the number of patients with abdominal perforations, destructive appendicitis and cholecystitis, as well as pancreatitis increases every year $[2,6]$.

\section{Corresponding Author:}

Vasyl Rybalchenko, MD, PhD, Professor of the Department of Pediatric Surgery at the P.L. Shupyk National Medical Academy of the Ministry of Health of Ukraine, Professor of the Department of Surgical Diseases No. 1 of the Kyiv Medical University of UAFM. E-mail: pedsurgery_ua@ukr.net
According to various authors, postoperative abscesses develop in $0.8-2 \%$ of operated patients, and mortality in these cases ranges from 10.5 to $26 \%$. Intra-abdominal abscesses and infiltrates rank second among the causes of repeated laparotomies in the early postoperative period. In case of inadequate treatment, mortality from this pathology reaches $45 \%$ and more $[2,6,7]$. The main causes of intra-abdominal abscesses and infiltrates development and mortality in acute appendicitis in Ukraine are the following: disease severity $19.7 \%$; late hospitalization - $46.1 \%$; technical mistakes during the operation $-5.2 \%$; tactical mistakes $-6.8 \%$; defects of postoperative treatment $-7.7 \%$; concomitant diseases $-9.3 \%$; late operation $-5.2 \%$. The problem has not been solved, and all complications and mortality rates indicate organizational problems and late referral $[1,6]$.

Thus, all of the above requires further study in order to predict the course of the disease and its adequate surgical treatment, for the sake of 
reducing both postoperative complications and mortality, which in general will improve patient's quality of life.

\section{Purpose, subjects and methods:}

2.1. The purpose: to improve the results of surgical treatment of patients with primary intraabdominal infiltrates, abscesses and fluid masses by introducing the latest innovative diagnostic and treatment technologies.

\subsection{Subjects \& Methods}

191 patients aged 16-85 (96 male (50.26\%) and 95 female $(49.74 \%)$ ) with primary intraabdominal infiltrates, abscesses and fluid masses were treated at the hospital of the Department of Surgical Diseases, at the Surgery Center of Kyiv City Clinical Hospital No. 1 from 2006 to 2019. Only the patients with primary fluffy infiltrates, abscesses and fluid masses were included in the study, and patients with dense infiltrates were not included in the study, because that group provided for conservative and then operative treatment. Depending on the time of hospitalization and use of diagnostic and therapeutic measures, the patients were divided into a control group - from 2006 to 2012 - 102 (53.40\%) patients and the study group - from 2013 to 2019 $89(46.6 \%)$ patients. General clinical and biochemical blood and urine tests were performed in all patients during hospitalization. Radiological examination (vertical and polypositional plan radiography of the abdominal cavity organs) was performed in $85(44.50 \%)$ patients. Ultrasound examination of the abdominal cavity organs was done in $78(40.84 \%)$ patients. Rectal and bimanual examination was performed in $100(52.36 \%)$ patients. Thermometry of the anterior abdominal wall was performed in $61(31.94 \%)$ patients.

\section{Results \& Discussion}

The patients were divided into 3 subgroups depending on the underlying disease; however, these were patients who were urgently hospitalized and had surgical treatment. The first group included 74 (38.74\%) patients with destructive appendicitis complicated by primary infiltrates and abscesses, of which $39(20.42 \%)$ control group patients and $35(18.32 \%)$ study group patients. The second group included $48(25.13 \%)$ patients with perforated gastric and duodenal ulcers, of which the control group was 26 $(13.61 \%)$, and the study group was $22(11.52 \%)$. The third group included 69 (36.13\%) patients with cholecystitis with primary complications in $69(36.13 \%)$ patients, including $37(19.37 \%)$ in the control group and $32(16.76 \%)$ in the study group.
First of all, it is reasonable to emphasize that the type of surgical treatment depended on the study group, since the study group included laparoscopic surgery and technology of tissue ligation and separation. Considering that each nosological unit is an independent disease, further percentage calculations were performed in each group.

Surgical treatment of $74(38.74 \%)$ patients with destructive appendicitis was complicated by primary infiltrates and abscesses differed in time of surgery. Thus, surgical access in 39 patients in the control group was laparotomy and was associated with the localization of infiltrative abscessed mass, of which $11(14.86 \%)$ had midline laparotomy and $28(37.84 \%)$ had right-sided transrectal laparotomy. However, among 35 (47.30\%) patients of the study group right-sided transrectal laparotomy was performed in $3(4.05 \%)$ of them, laparoscopy in $20(27.03 \%)$ and diagnostic laparoscopy with conversion in $12(16.22 \%)$ patients. The reasons for conversion during surgery were: beginning of the introduction of laparoscopic techniques and subhepatic, retroperitoneal location of the appendix in $5(6.76 \%)$ patients and in the pelvic cavity in $1(1.35 \%)$ patient, which made it impossible to separate it. Separation of the infiltrative-abscess formation in the control group was purely mechanical using instruments and cotton swab, and hemostasis was performed using monopolar coagulation and tissue piercing. On the other hand, in the control group of $35(47.40 \%)$ patients, a water-jet device for tissue preparation was used in $15(20.27 \%)$ patients to separate infiltrate-abscess formations, as well as bipolar coagulation and intracorporeal suturing for the purpose of hemostasis. During laparoscopy, infiltration-abscess formations were separated in $20(27.03 \%)$ patients using bipolar coagulation. The next stage of surgery differed depending on the successful separation of the infiltrative-abscess formations. During the separation of formations in $39(54.6 \%)$ patients in the control group, staged resection of the greater omentum with vascular stitching (non-resorbable thread), as well as monopolar coagulation of blood vessels were used. In contrast, bipolar coagulation of the greater omentum and soft tissues was used in $35(47.40 \%)$ patients of the control group, of which in 15 (20.27\%) patients openly, and in 20 $(27.03 \%)$ by laparoscopy. Separation and mobilization of the appendix and the mesentery was performed as follows. In the control group, the appendix and the mesentery were separated, and then non-resorbable sutures were applied, of which $25(33.78 \%)$ patients had two sutures. The 
stump of the appendix was tied and implanted under the cisternal and Z-shaped sutures in 23 $(31.08 \%)$ patients, and in $16(21.62 \%)$ other patients, separate stump invaginations in number from 6 to 8 were placed because of a pronounced inflammatory process (suture cutting out). In the study group, the mesentery of the appendix was sutured with bipolar in $20(27.03 \%)$ patients, stapler and Z-shaped sutures were applied to the base of the appendix in $20(27.03 \%)$ patients. Debridement of the abdominal cavity was of great importance. Thus, in the control group we performed debridement with antiseptics, and in the study group we used physiological solution until complete removal of purulent contents and fibrin. Later there was a question of abdominal cavity drainage, and single-lumen (control group) and doublelumen (study group) drains were used, and the number of drains was determined individually depending on the spread of inflammatory process. However, we consider it necessary to arrange the drainage through a separate access, which was performed in $35(47.40 \%)$ patients of the control group and in $12(16.22 \%)$ patients of the study group. Pelvic cavity drainage was performed in $46(62.16 \%)$ patients, which allowed avoiding postoperative complications. The following complications were found in the postoperative period: postoperative wound infiltrate in 14 $(18.92 \%)$ patients, postoperative wound seroma in $9(12.16 \%)$ patients, suppuration of the wound channel in $3(4.05 \%)$ patients control group). The drains were removed in the absence of discharge.

Surgical treatment of $48(25.13 \%)$ patients with perforated gastric and duodenal ulcers who had infiltrative-abscessing complications of greater omentum. Surgical access in all 48 patients was made by midline laparotomy with revision and separation of the perforated-inflammatory process caused by the action of hydrochloric acid and bile on the peritoneal walls. Localization of perforated gastric ulcer: $5 \mathrm{~cm}$ to the duodenal bulb in 7 $(14.58 \%)$ patients, closer to the small curvature in $12(25.0 \%)$ patients, at the bottom of the stomach in $14(29.17 \%)$ patients, in the center of the stomach in $9(18.75 \%)$ patients, at the level of duodenal bulb in $10(20.83 \%)$ patients, the anterior part of the duodenum in $3(6.25 \%)$ patients, the lower part of the duodenum in 5 $(10.42 \%)$ patients. Surgical differences between the control and study groups consisted in the use of vicryl sutures instead of Capron ones, as well as the use of mono and bipolar for hemostasis. Mobilization and separation of the infiltrated greater omentum with resection and stitching in the control group in contrast to the study group using water-jet scalpel in 18 (37.5\%) patients for precise "bloodless" tissue separation. In all patients the perforated ulcer margins were cleaned of callous tissues and double-row sutures "resorbable sutures with an interval of more than 40 days" were applied against a background of the probe in the stomach and duodenum, with mandatory control of the tightness of the sutures. After the defect was eliminated, the abdominal cavity was examined, washed and drained. In the study group, two-lumen drainages were placed both to the perforation site and the pelvic cavity. In the postoperative period, the postoperative wound infiltrate was diagnosed in $2(4.17 \%)$ patients in the study group, where the conservative treatment was effective and the postoperative wound seroma in $3(6.25 \%)$ patients in the control group, where the removal of one suture (debridement and drainage) treatment was effective. Drainage in all patients was performed through a separate access, and removal was performed when there was no discharge and peristalsis was restored on the $3^{\text {rd }}-5^{\text {th }}$ day. Suture failure was not detected in the control and study groups.

Surgical treatment of $69(100 \%)$ patients with cholecystitis had primary infiltrative abscessed complications that differed by groups. Thus, in the control group $37(53.62 \%)$ patients had the laparotomy access along the costal arch, in 32 $(46.38 \%)$ patients of the study group, the access was performed by laparotomy in $6(8.69 \%)$ of them, laparoscopy in $26(37.68 \%)$ patients, of which $5(7.25 \%)$ underwent conversion due to a pronounced sclerotic adhesions of the greater omentum and the walls of the large intestine. The greater omentum was involved in the infiltrativeabscess formation of all 69 patients, as well as the walls of the large intestine (transverse colon) in $18(26.08 \%)$ patients. In $37(53.62 \%)$ patients of the control group, the infiltrative-abscess process was separated mechanically, and in 11 (15.94\%) patients of the study group, water-jet device was used. Mobilization and resection of the greater omentum was performed by stitching the area of the mobilized omentum with nonresorbable threads in 37 (53.62\%) patients, using bipolar in $32(46.38 \%)$ patients of the study group. Biliary bile extraction in patients of the control group was carried out using monopolar, which led to certain difficulties and bleeding, and in the patients of the study group - using bipolar, which allowed performing almost bloodless surgical intervention. Laparoscopic cholecystectomy was performed in $21(30.43 \%)$ patients using bipolar 
coagulation. In the postoperative period, drainage of the abdominal cavity was performed, with the difference that in the study group it was performed by double-lumen drainage. In the postoperative period, the postoperative wound suppuration occurred in $2(2.90 \%)$ patients of the control group and in one $(1.4 \%)$ patient of the study group.

Thus, primary intra-abdominal abscesses and infiltrates were associated with the organ, of which destructive appendicitis was found in 74 (38.74\%) patients, perforated gastric and duodenal ulcers in $48(25.13 \%)$ patients, cholecystitis in $69(36.13 \%)$ patients. Visualization of primary and secondary disease was based on ultrasound in $78(40.84 \%)$, abdominal and thoracic radiological examination in $85(44.50 \%)$, anterior abdominal wall thermometry in $61(31.94 \%)$, and rectal examination in $100(52.36 \%)$ patients. Surgical treatment was individualized depending on the disease. In destructive appendicitis of 74 $(38.74 \%)$ patients, laparotomy was performed in $42(21.99 \%)$ patients, laparoscopic procedure in $32(16.75 \%)$, and in $12(6.28 \%)$ patients with conversion. $48(25.13 \%)$ patients perforated gastric and duodenal ulcer underwent open laparotomy. Of $69(36.13 \%)$ patients with cholecystitis, $48(25.13 \%)$ underwent laparotomy and $21(11.00 \%)$ - laparoscopy. The use of the recent water-jet technique in $64(33.51 \%)$ patients to separate infiltrative abscesses allowed minimizing the damage to the serous membrane and cleaning the peritoneum from the acquired formations. The control group demonstrated better results of surgical treatment, taking into account the quality of life.

Surgical treatment of intra-abdominal abscesses and infiltrates is always controversial with regard to diagnostic methods, that is, imaging of the disease, surgical treatment methods, as well as drainage and use of suture material, and antibacterial therapy aimed at stopping the spread of infection.

Among acute surgical diseases of the abdominal cavity, acute appendicitis (AA) accounts for $89.1 \%$, ranking first in Ukraine. Appendectomy accounts for about $20-30 \%$ of all surgical procedures. Based on the protocol, the proportion of patients hospitalized later than 24 hours from the onset of the disease ranges from 4.9 to $31.2 \%$ and averages $20.9 \%$ in the country, and postoperative mortality among them is $0.15 \%(0$ to $0.4 \%$ ) [1]. It is known that the incidence of AA has been steadily decreasing since the late 1940s. Thus, in developed countries, AA occurs in 5.7-50 patients per 100,000 inhabitants per year, with a peak between the ages of 10 and 30 years. Geographic differences have also been established: the lifetime risk of AA is $9 \%$ in the United States, $8 \%$ in Europe, and 2\% in Africa. Moreover, there are large differences in clinical manifestations, disease severity, radiological examination, and surgical treatment of patients with AA, which are related to the income of the country [10].

According to the EAES (European Association for Endoscopic Surgery) summary regarding the diagnosis of AA, diagnostic imaging can reduce the negative appendectomy rate, which reaches $15 \%$. Ultrasound, abdominal computed tomography (CT) and magnetic resonance imaging (MRI) are most commonly used methods. Ultrasound has a sensitivity of 71 to $94 \%$ and a specificity of 81 to $98 \%$. The incidence of purulentseptic complications after appendectomy ranges from 0 to $11 \%$, and other complications (stump suture failure, adhesive obstruction) range from 3.0 to $28.7 \%$ [25].

Thus, according to Di Saverio et al. (2020), the incidence of appendix perforation ranges from $16 \%$ to $40 \%$, with a higher incidence in younger age groups $(40-57 \%)$ and in patients over 50 years of age ranging from 55 to $70 \%$. The risk of death from non-gangrenous appendix is less than $0.1 \%$, but the risk increases to $0.6 \%$ in gangrenous appendix. On the other hand, perforated appendix has a higher mortality rate of about $5 \%$ [10]. The same authors established the role of diagnostic imaging, such as ultrasound, computed tomography (CT) or magnetic resonance imaging (MRI) in the diagnosis of the disease [10].

Further studying the diagnosis of destructive appendicitis (DA), Matthew Fields J. et al. (2020) found that sensitivity and specificity of ultrasound is $91 \%$ and $97 \%$ respectively with a positive and negative predictive value of $91 \%$ and $94 \%$ respectively [21]. A meta-analysis by Duke E. et al. (2016) on the use of MRI in the diagnosis of appendicitis during pregnancy found a sensitivity of $90.5 \%, 94 \%$ and $91.8 \%$; specificity of $98.6 \%$, $97 \%$ and $97.9 \%$ respectively, and a positive predictive value of $86.3 \%$ and a negative predictive value of $99.0 \%$ [12].

In order to diagnose infiltrative inflammatory appendicular complications of abscesses and infiltrates, Rybalchenko V.F., Demidenko Yu.G. (2016) used infrared thermometry of the anterior abdominal wall and self-developed axillary pain factor in order to interpret the increased temperature. In all observations, the method resulted to be informative [5]. 
The issue of both appendix extraction and suture fusion is still relevant today. Diamantis T. et al. (2006) compared LigaSureTM and Harmonic Scalpel with monopolar electrocoagulation and bipolar coagulation: the first two had minimal thermal tissue damage than other methods [11]. However, studies by Pogorelic Z. found higher thermal damage of the mesoappendix and its base in patients during surgery using LigaSureTM than in patients using Harmonic Scalpel [23]. The studies by Skyba V.V. et al. (2017) proved that the use of water-jet scalpel to separate infiltrative inflammatory process and mobilize the appendix is a bloodless method [6].

The treatment of the mesentery and the base of the appendix is an important problem, since isolated cases of appendix ligature failure have been described in the literature. Thus, Wright G.P. et al. (2015) suggested the use of a single stapler line to dissect the mesoappendix and apply to the appendix as a safe and effective method that leads to a shorter duration of surgery and excellent surgical results [31]. A meta-analysis by Antoniou S.A. et al. (2017) involving more than 5000 patients showed that the use of suture material, that is, appendix ligation is superior to other methods given the combined parameters of infection of the organ and the surface of the operating field [8]. In another study, Qian D. et al. (2015) compared simple ligation and stump invagination, and no significant difference was found, and as a consequence, clinical results showed that simple ligation was significantly superior to stump invagination [24]. Abdominal drainage is also debated. According to the retrospective study by Schlottmann F. et al. (2016), the placement of intra-abdominal drainage in complicated appendicitis did not bring benefits in terms of reducing the infectious process, and was characterized by an increase in the duration of hospital stay [26].

Regarding the development of cholecystitis, it has been established that in $10-15 \%$ of patients, gallbladder wall ischemia develops against a background of intravesical hypertension, which leads to necrosis, perforation and peritonitis. The most frequent forms are perivesical (subhepatic, subdiaphragmatic) abscesses. In gas-forming flora, emphysematous gallbladder is more common. Ultrasound allows to differentiate between acute obstructive (catarrhal) and acute destructive cholecystitis and distinguish gangrenous cholecystitis and establish gallbladder wall integrity disorder and peripapillary abscess [3]. According to Kimura Y. (2013), Pisano, M. (2020), 20 to $40 \%$ of patients with gallbladder stones develop gallbladder stone-related complications with an incidence of $1-3 \%$ annually [17]. In order to verify the disease, Gurusamy et al. (2015) performed a meta-analysis of ultrasound verification with a sensitivity of $95 \%$ and specificity of $95 \%$ [15].

Cholecystectomy is the most common approach and is considered the standard of care for gallstone disease for most patients. The causes of gallbladder obstruction can vary and may be related to obesity, adhesions, acute or chronic inflammation, gallbladder bloating and cirrhosis. Options include subtotal cholecystectomy [16]. A meta-analysis by Elshaer et al. (2015) showed that subtotal cholecystectomy was performed using laparoscopic $(72.9 \%)$ open $(19.0 \%)$ and laparoscopic, convertible to open (8.0\%) techniques. The number of patients was more than 1200 , and the most frequent indications were severe cholecystitis (72.1\%), cirrhosis and portal hypertension $(18.2 \%)$ and empyema or perforated gallbladder (6.1\%). [13].

Conservative treatment of acute cholecystitis is relevant, and therefore Schmidt M. (2011) with long-term observation for 14 years approximately $30 \%$ of patients who received conservative treatment developed recurrent complications associated with gallstone disease, and $60 \%$ of patients who underwent cholecystectomy [27].

Postoperative complications are more frequently diagnosed in men, ranging from 10 to $15 \%$, with increased conversion to open cholecystectomy from 16 to $48.5 \%$ and, according to the author, are associated with increased skeletal muscle mass [28]. Meanwhile, Campanile F.C. (2014) presented hospital mortality and cholecystostomy incidence ranging from 4 to $50 \%$ and from 8.2 to $62 \%$, respectively [9].

Perforated gastric and duodenal ulcers are clinically manifested by sudden abdominal pain with the development of localized or generalized peritonitis and may be present in only two-thirds of patients $[29,30]$. In terms of diagnosis, the first diagnostic test is an abdominal and chest X-ray to determine the presence of free air in the abdomen and varies widely among studies reported in the literature, ranging from 30 to $85 \%$. Meanwhile, a negative $\mathrm{X}$-ray does not exclude a possible perforation, and therefore a CT scan is advisable $[14,30]$.

The work of Lee F.Y. (2001) is of scientific and practical significance, involving 374 patients who had surgical treatment for perforated ulcer disease, of whom 219 patients received open treatment (suturing), 109 patients received laparoscopic treatment with fibrin glue, and the remaining 46 patients were treated with laparoscopic 
suture. At the beginning of treatment, laparoscopic fibrin glue plastics were performed on 149 patients, but 40 had to be transferred to suturing. The overall conversion rates for laparoscopic fibrin glue plastics and laparoscopic suture plastics were 27 and $15 \%$, respectively. The main reasons for conversion were a large ( $1 \mathrm{~cm}$ or more) perforated ulcer, as well as the inability to determine the location of the perforation. Mean-while, the overall rate of leaks after laparoscopic glue plastics and laparoscopic suture plastics was 16 and $6 \%$, respectively, and the rate of reope-ration for clinical leaks after laparoscopic glue plastics and laparoscopic suture plastics was 10 and $4 \%$, respectively [ 18 , 30]. Instead, studies by Lin et al. (2017) analyzed the surgical treat-ment of 118 patients with perforated ulcers who underwent laparoscopic plastics with simple suturing $(\mathrm{n}=27)$ and omentopexy $(\mathrm{n}=91)$, and found three failures of sutures after closure: 1 after simple closure and 2 after closure and omento-pexy, but no patient died [19].

Based on the results of treatment, an analysis of the Massimo S. at al. [2017] was studied, as well as multicenter observation conducted in 132 medical institutions around the world over a 4-month period (October 2014 - February 2015), and included 4553 patients who had intra-abdominal infection. According to the results of the study, the established overall mortality was $9.2 \%$ [20].
In conclusion, it is worth emphasizing that the diagnosis and adequate treatment of primary infiltrative and abscessing formations, despite the achievements, remains an extremely difficult problem.

\section{Conclusions}

1. The frequency of primary intra-abdominal complications in the form of infiltrates and abscesses is associated with anatomical localization: most often it is destructive appendicitis, to a lesser extent destructive cholecystitis and perforated gastric ulcer and duodenal ulcer, and among postoperative it is adhesive obstruction and hernia strangulation.

2. Visualization of primary and postoperative secondary intra-abdominal complications is based on a comprehensive examination with the account the informativity and safety: thermometry of the anterior abdominal wall, ultrasound, X-ray examination of the abdominal cavity and rectal examination of the patients.

3. The use of a water-jet scalpel and bipolar coagulation allows precise and bloodless separation of infiltrative-abscessing formations, which yields better results of surgical treatment, taking into account the quality of life.

Conflict of interests

The authors of the article declare no conflict of interests.

\section{References}

1. Acute appendicitis. (2016) Unified clinical protocol of emergency, primary and secondary (specialized) medical care. Ministry of Health of Ukraine. Kyiv, 2016. 75 p.

2. Demyanova V.N. (2015) Optimization of minimally invasive methods of treatment of intra-abdominal abscesses. author's abstract...of Ph.D.: 14.01.17 - Stavropol Surgery - 2015. 25 p.

3. Dolimov K.S., Ilkhamov F.A. (2014) Gallbladder infiltrate. Tashkent Pediatric Medical Institute of the Republic of Uzbekistan // Clinical Surgery. 2014;3:23-24.

4. Zaremba E.H., Zaremba V.S., Rak N.A., Girniak A.T., Zaremba O.V., Burmay S.V. (2020) Perivesical gallbladder infiltrate with extension to the subhepatic space (a clinical case). Practicing Physician. 2020;34:10-15.

5. Rybalchenko V.F., Demidenko Yu.G. (2019) Thermometric panel of the anterior abdominal wall and prognostic axillary pain factor. / Neonatology, Surgery, and Perinatal Medicine. 2019. т.IX, No. 3.C.86-94. Doi: https://doi.org/10.24061/2413-4260.IX.3.33.2019.4

6. Skyba V.V., Rybalchenko V.F., Ivanko O.V., Demidenko Yu.G., Badakh V.M., Bocharov V.P. (2017) Surgical treatment of inflammatory and adhesive processes of the abdominal cavity in adolescents using a water-jet scalpel. // Child Health2017.volume. 12. No.1., p. 68-74. http://nbuv.gov.ua/UJRN/ Zd_2017_12_1_14

7. Fomin D., Usenko A.Yu., Bereznitskii Y.S. (2018) Emergency surgery of abdominal cavity organs (organization standards and professionally oriented algorithms of medical care). Kyiv: Library "Health of Ukraine", 2018. - 354 p.

8. Antoniou SA, Mavridis D, Hajibandeh S, et al. (2017) Optimal stump management in laparoscopic appendectomy: A network meta-analysis by the Minimally Invasive Surgery Synthesis of Interventions and Outcomes Network. Surgery. 2017;162:994-1005. DOI: 10.1016/j.surg.2017.07.013

9. Campanile FC, Pisano M, Coccolini F, et al. (2014) Acute cholecystitis: WSES position statement. World J Emerg Surg. 2014;9(1):1-6. DOI https://doi.org/10.1186/1749-7922-9-58 
10. Di Saverio et al. (2020) Diagnosis and treatment of acute appendicitis: 2020 update of the WSES Jerusalem guidelines. World Journal of Emergency Surgery (2020) 15:27 https://doi.org/10.1186/s13017020-00306-3

11. Diamantis T, Kontos M, Arvelakis A, et al. (2006) Comparison of monopolar electrocoagulation, bipolar electrocoagulation, Ultracision, and Ligasure. Surg Today. 2006;36:908-13.

12. Duke E, Kalb B, Arif-Tiwari H, et al. (2016) A systematic review and meta-analysis of diagnostic performance of MRI for evaluation of acute appendicitis. Am J Roentgenol. 2016;206:508-17.

13. Elshaer M, Gravante G, Thomas K, Sorge R, Al-Hamali S, Ebdewi H. (2015) Subtotal cholecystectomy for "Difficult gallbladders": Systematic review and meta-analysis. JAMA Surg. 2015;150(2):159-68.

14. Grassi R, Romano S, Pinto A, Romano L. (2004) Gastro-duodenal perforations: conventional plain film, US and CT findings in 166 consecutive patients. Eur J Radiol. 2004;50:30-6.

15. Gurusamy K, Giljaca V, Takwoingi Y, et al. (2015) Ultrasound versus liver function tests for diagnosis of common bile duct stones. Cochrane Database Syst Rev. 2015;2:CD011548.

16. Hussain A. (2011) Difficult laparoscopic cholecystectomy: Current evidence and strategies of management. Surg Laparosc Endosc Percutaneous Tech. 2011;21(4):211-7.

17. Kimura Y, Takada T, Strasberg SM, et al. (2013) TG13 current terminology, etiology, and epidemiology of acute cholangitis and cholecystitis. J Hepatobiliary Pancreat Sci. 2013;20(1):8-23.

18. Lee FY, Leung KL, Lai PB, Lau JW. (2001) Selection of patients for laparoscopic repair of perforated peptic ulcer. Br J Surg. 2001;88:133-6.

19. Lin BC, Liao CH, Wang SY, Hwang TL. (2017) Laparoscopic repair of perforated peptic ulcer: simple closure versus omentopexy. J Surg Res. 2017;220:341-5.

20. Massimo Sartelli, Alain Chichom-Mefire, Fausto Catena. (2017) The management of intra-abdominal infections from a global perspective: 2017 WSES guidelines for management of intra-abdominal infections. World Journal of Emergency Surgery volume 12, Article number: 29 (2017), 12:36

21. Matthew Fields J, Davis J, Alsup C, et al. (2017) Accuracy of point-of-care ultrasonography for diagnosing acute appendicitis: a systematic review and meta-analysis. Acad Emerg Med. 2017;24:1124-36.

22. Pisano, M., Allievi, N., Gurusamy, K. et al. (2020) 2020 World Society of Emergency Surgery updated guidelines for the diagnosis and treatment of acute calculus cholecystitis. World J Emerg Surg 15, 61 (2020). https://doi.org/10.1186/s13017-020-00336-X

23. Pogorelic Z, Katic J, Mrklic I, et al. (2017) Lateral thermal damage of mesoappendix and appendiceal base during laparoscopic appendectomy in children: comparison of the harmonic scalpel (Ultracision), bipolar coagulation (LigaSure), and thermal fusion technology (MiSeal). J Surg Res. 2017;212: 101-7.

24. Qian D, He Z, Hua J, et al. (2015) Stump invagination versus simple ligation in open appendicectomy: a systematic review and meta-analysis. Int Surg. 2015;100:1199-206.

25. Ramon R. Gorter, Hasan H. Eker, Jaap Bonjer. (2015) Diagnosis and management of acute appendicitis. EAES consensus development conference 2015. Surgical Endoscopy volume 30, pages 4668-4690 (2016) https://link.springer.com/article/10.1007/s00464-016-5245-7\#citeas

26. Schlottmann F, Reino R, Sadava EE, et al. (2016) Could an abdominal drainage be avoided in complicated acute appendicitis? Lessons learned after 1300 laparoscopic appendectomies. Int J Surg. 2016;36:40-3.

27. Schmidt M, Sondenaa KVM, et al. (2011) Long-term follow-up of a randomized controlled trial of observation versus surgery for acute cholecystitis: non-operative management is an option in some patients. Scand J Gastroenterol. 2011;46:1257-62.

28. Schweitzer L, Geisler C, Pourhassan M, et al. (2016) Estimation of Skeletal Muscle Mass and Visceral Adipose Tissue Volume by a Single Magnetic Resonance Imaging Slice in Healthy Elderly Adults. J Nutr. 2016;146(10):2143-8.

29. Soreide K, Thorsen K, Soreide JA. (2014) Strategies to improve the outcome of emergency surgery for perforated peptic ulcer. Br J Surg. 2014;101:e51-64.

30. Tarasconi, A., Coccolini, F., Biffl, W.L. et al. (2020) Perforated and bleeding peptic ulcer: WSES guidelines. World J Emerg Surg 15, 3 (2020). https://doi.org/10.1186/s13017-019-0283-9

31. Wright GP, Mitchell EJ, McClure AM, et al. (2015) Comparison of stapling techniques and management of the mesoappendix in laparoscopic appendectomy. Surg Laparosc Endosc Percutan Tech. 2015; 25: e11-5. doi: 10.1097/SLE.0000000000000040.

Received: 29-Nov-2020 Accepted: 11-Mar-2021 